\title{
Aluminium-Doped Zinc Oxide Rear Reflectors for High-Efficiency Silicon Heterojunction Solar Cells
}

\author{
Laurie-Lou Senaud, Gabriel Christmann, Antoine Descoeudres, Jonas Geissbühler, Loris Barraud, \\ Nicolas Badel, Christophe Allebé, Sylvain Nicolay, Matthieu Despeisse, \\ Bertrand Paviet-Salomon, and Christophe Ballif
}

\begin{abstract}
This contribution demonstrates an improved infrared response of the rear reflector of monofacial silicon heterojunction solar cells using aluminium-doped zinc oxide (AZO) in lieu of indium tin oxide (ITO) in the back electroncollecting shell. Along these lines, the carrier concentration and the thickness of the rear AZO layer are optimized in order to minimize the free-carrier and the plasmonic absorption losses without detrimentally affecting the selectivity and the electrical transport properties of the device. The respective reductions of free-carrier vs. plasmonic absorption losses are thoroughly analyzed. Furthermore, the open-circuit voltage and series resistance of the solar cells are shown to not be impacted by the AZO thickness and the carrier concentration within the investigated ranges. As a result of these optimizations, a significant decrease in the parasitic absorption is obtained, leading to a champion device with a short-circuit current density of up to $40.81 \mathrm{~mA} / \mathrm{cm}^{2}$ and an efficiency of $23.96 \%$, featuring a standard screen-printed silver grid at the front with ca. $3.25 \%$ optical shadowing. In summary, AZO appears to be a promising indium-free alternative material to replace the back ITO commonly used in silicon heterojunction solar cells.
\end{abstract}

Index Terms-Aluminium-doped zinc oxide, electrical transport, heterojunction, optical absorption, rear reflector, silicon, solar cell.

\section{INTRODUCTION}

$S^{n}$ ILICON heterojunction (SHJ) solar cells consist of different thin material layers deposited on both sides of a crystalline silicon (c-Si) bulk absorber. These layers have the function of extracting the photo-generated carriers and injecting them into an external load without detrimentally impeding the light absorption in the absorber. These different layers typically consist of hydrogenated amorphous silicon (a-Si:H), transparent conductive oxide (TCO) and metal, which together have to ensure the electrical roles of: passivation, carrier selectivity and electrodes. In a recently revisited framework, layer stacks fulfilling these requirements have been described as selective membranes [1] or skins [2], [3], [4]. Similarly to these terminologies, the term shell will be used throughout this paper to refer to the whole a-Si:H/TCO/metal stack. The excellent full area passivation level of SHJ shells, coupled with high c-Si bulk quality, enables open-circuit voltage $\left(\mathrm{V}_{\mathrm{OC}}\right)$

L.-L. Senaud, G. Christmann, A.Descoeudres, J. Geissbühler, L. Barraud, N. Badel, C. Allebé, S. Nicolay, M. Despeisse, B. Paviet-Salomon, and C. Ballif are with the CSEM, PV-Center, Rue Jaquet-Droz 1, CH-2002 Neuchâtel, Switzerland (e-mail: laurie-lou.senaud@csem.ch, gabriel.christmann@csem.ch, values of up to $750 \mathrm{mV}$ [5]. Despite these high $\mathrm{V}_{\mathrm{OC}}$ values, efficiency is still limited by the optical losses due to the parasitic absorption of light from the front and the rear shells [6], [7]. In particular, in the rear shell of monofacial SHJ solar cells the TCO and metal reflector, if not properly optimized, are the origin of significant losses in the infrared (IR) part of the spectrum. These losses come from plasmonic absorption at the $\mathrm{TCO} /$ metal interface and from free-carrier absorption (FCA) in the TCO [6], [8]. Reducing these IR parasitic absorption losses constitutes an important challenge in the quest to improve the device short-circuit current density $\left(\mathrm{J}_{\mathrm{SC}}\right)$ and therefore, the SHJ device efficiency (Eff.). Plasmonic absorption is mainly induced by the parallel-polarized light of evanescent waves impinging on the metal surface which excites surface plasmons [8]. In addition, this absorption has been demonstrated to depend on the metal type and on its surface roughness [9]. FCA in contrast is induced by the free carriers contained inside the TCO, which mainly absorb light in the IR part of the solar spectrum. According to the Drude model, the FCA is directly proportional to the carrier density and inversely proportional to the optical mobility of the material [10]. Both parameters depend on oxygen vacancies in TCO [11] which can be modified by varying the oxygen partial pressure during TCO deposition [12]. An increase in free carrier density induces a shift of the IR absorption edge to shorter wavelengths, thus narrowing the transmission window. This shift is determined by the plasma oscillation of the free carriers, which screen, for IR energy, the incident electromagnetic wave via intra-band transitions within the conduction band. This involves a sharp reduction of the optical transmission near the plasma resonance frequency. Thus any attempt to decrease the resistivity of the TCO by increasing the carrier density will increase the IR light absorption. As the main part of the solar spectrum reaching the rear side of the SHJ solar cell actually consists of IR light, back reflectors may suffer then from both of the above-mentioned absorption mechanisms. To reduce the plasmonic rear absorption, a moderately absorbing 150 to 200 -nm-thick TCO layer is usually deposited on the back of SHJ solar cells [13], [14]. This thick layer reduces the amount of evanescent waves reaching the metal by increasing the internal reflectance at the rear surface, which yields to an increase of the IR spectral

jonas.geissbuehler@csem.ch, nicolas.badel@csem.ch,

loris.barraud@indeotec.com, christophe.allebe@csem.ch sylvain.nicolay@csem.ch, matthieu.despeisse@csem.ch, bertrand.pavietsalomon@csem.ch, christophe.ballif@csem.ch). 
response of the cell [6]. Such a structure with a thick back TCO efficiently reduces the plasmonic absorption in the metal. However, as the TCO layer becomes thicker, the FCA losses will become more and more important (for given material properties). Therefore, care must be taken not to negate the optical gains provided by the reduction of the plasmonic absorption losses with an increase in FCA when thickening the TCO layer. To get around this trade-off, one alternative option is to partly replace the rear TCO with a transparent dielectric layer such as magnesium fluoride [7]. Even if this approach convincingly demonstrated reduced optical losses with limited detrimental impact on FF and $\mathrm{V}_{\mathrm{OC}}$ [7], [15], it did not yet demonstrate a better IR response than a well-optimized full TCO rear reflector - such as the one presented in this paper and requires additional processing steps. In addition, as the rear TCO layer has to efficiently ensure the electrical contact between the rear a-Si:H and metal layers, several constraints on the electrical properties of the former are present. Therefore, the degrees of freedom available to tune it are reduced. Furthermore, the TCO properties, and particularly its Fermi level position at the a-Si:H interface (or, as often used equivalently, its workfunction) are known to strongly affect the SHJ cell selectivity and transport losses. In the case of an electron-collecting shell, a low workfunction TCO - and hence a high carrier concentration - is required to obtain a favorable band alignment enabling high fill factor (FF) [16],[17]. There is therefore a tradeoff between the optical and the electrical properties of the SHJ rear shell; all its components need to be considered together to improve the final performances of the device ${ }^{1}$.

Along these lines, this contribution aims at improving the back IR reflector of monofacial SHJ solar cells by mitigating its optical losses without compromising the transport properties of the device, i.e its $\mathrm{V}_{\mathrm{OC}}$ and FF, using an indium-free TCO. In SHJ solar cells, aluminum-doped zinc oxide (AZO) has already demonstrated promising performance. As a back TCO in the rear electron-collecting shell, AZO has demonstrated Eff. of up to $22.9 \%$ with high electrical contact performances [18]. In addition, using AZO in front electron-collecting shells, devices with Eff. up to $23 \%$ were demonstrated along with a $\mathrm{J}_{\mathrm{SC}}$ gain of $0.5 \mathrm{~mA} / \mathrm{cm}^{2}$ compared to a reference cell featuring an indium tin oxide (ITO) layer [19]. In the present work, AZO is further optimized as an indium-free alternative material to the standard back ITO. Similarly to what was done in [6] for ITO, the thickness of the rear AZO layer and the oxygen $\left(\mathrm{O}_{2}\right)$ content in the deposition gas flow are varied to find the optimal combination leading to the highest $\mathrm{J}_{\mathrm{SC}}$. The related impacts on the device selectivity and the transport losses are also investigated. As a result of these optimizations, a $\mathrm{J}_{\mathrm{SC}}$ of up to $40.81 \mathrm{~mA} / \mathrm{cm}^{2}$ is obtained without detrimental effects on the $\mathrm{V}_{\mathrm{OC}}$ and the FF, leading to Eff. of up to $23.96 \%$ for two sidecontacted SHJ devices with front screen-printed contacts.

\footnotetext{
${ }^{1}$ For the sake of completeness, note that the same kind of optical and electrical constraints also apply to the front TCO. Indeed, the front TCO is the conductive layer for the lateral current flowing to the screen-printed silver grid and serves also as an antireflective coating to minimize light reflection. In
}

\section{EXPERIMENTAL DETAILS}

Silicon heterojunction solar cells with front hole-collecting shells were fabricated on 4 inch, $3 \Omega \cdot \mathrm{cm}$, n-type float zone c-Si wafers. Figure 1 shows a schematic description of the SHJ solar cell architecture under investigation. The wafers were textured on both sides in an alkaline solution to create random pyramids, resulting in a final wafer thickness of $240 \mu \mathrm{m}$ which was then wet chemically cleaned. The native silicon oxide was removed in a diluted hydrofluoric solution. Thin blanket intrinsic a-Si:H films (a-Si:H(i)) were then deposited on both sides of the wafers by plasma-enhanced chemical vapor deposition (PECVD) for chemical passivation. Then, thin blanket $\mathrm{p}$ - and n-type a-Si:H films (a-Si:H(p) and a-Si:H(n), respectively) were immediately deposited in the same deposition system to create the hole and the electron selective contacts at the front and at the rear side, respectively. More details on our a-Si:H stacks can be found elsewhere [20], [21]. The thicknesses of the a-Si:H layers, measured on planar glass substrates by spectroscopic ellipsometry, are typically $\sim 10 \mathrm{~nm}$. The implied- $\mathrm{V}_{\mathrm{OC}}\left(\mathrm{iV_{ \textrm {OC } }}\right)$ measured by the photoconductance decay method in transient mode [22] was around $730 \mathrm{mV}$ for all the samples of this study. An ITO layer was then deposited at the front side of the wafers using DC magnetron sputtering with an $\mathrm{In}_{2} \mathrm{O}_{3}: \mathrm{SnO}_{2}$ target. A metallic shadow mask was used during the front ITO deposition to define five $4-\mathrm{cm}^{2}$ solar cells per wafer. The front ITO thickness measured on planar bare glass substrates was $110 \mathrm{~nm}$ corresponding to an optimized antireflective coating. At the back side of the devices, AZO was deposited by RF magnetron sputtering with an Evatec Clusterline tool using different $\mathrm{O}_{2}$ contents in the deposition gas flow and deposition times, leading to different carrier concentrations and layer thicknesses as listed in Table I. The AZO layer thickness was assessed by a stylus profilometer on bare planar glass witness samples. The carrier density, the electrical mobility and the resistivity were measured by Hall effect measurement in the Van der Pauw configuration [23] on planar glass samples with a-Si:H(i)/a$\mathrm{Si}: \mathrm{H}(\mathrm{n})$ pre-deposited layers. Finally, a 200-nm-thick silver rear blanket layer was sputtered over the whole back AZO layer and a silver front grid was screen-printed on the front ITO of each cell. After this step, wafers were annealed at $210{ }^{\circ} \mathrm{C}$ for 30 minutes to cure the low-temperature printed silver paste. It is important to note that thanks to our large-area PECVD and PVD tools, within a single experiment, all wafers were codeposited with identical a-Si:H, front ITO and rear blanket silver layers. Therefore, only the screen-printing and each specific AZO deposition were performed individually for each wafer. 1-sun current-voltage (I-V) measurements were performed on each completed cell under Air Mass 1.5G equivalent illumination at $25^{\circ} \mathrm{C}$ using a Wacom class AAA light simulator. From these I-V curves, the cell parameters $\mathrm{J}_{\mathrm{SC}}$, $\mathrm{V}_{\mathrm{OC}}, \mathrm{FF}$ and Eff. were extracted. The series resistance $\left(\mathrm{R}_{\mathrm{S}}\right)$ values of each cell were calculated with the method presented

addition, the sheet resistance of the front TCO depend directly on the metal grid pitch and must therefore be optimized taking into account the latter. The front TCO is then also an important source of FCA losses, yet with less degrees of freedom to optimize it compared to the rear reflector [6]. 
in [24] using the 1-sun IV curve and a shaded IV curve with an illumination corresponding to about $10 \%$ of 1 -sun. In addition, the pseudo-fill factor $(\mathrm{pFF})$ was determined from pseudo-IV curves built from I-V curves measured at different illumination levels [25]. Finally, external quantum efficiency (EQE) and reflectance $(\mathrm{R})$ curves were measured directly on the cells with a PV-Tools Loana setup.

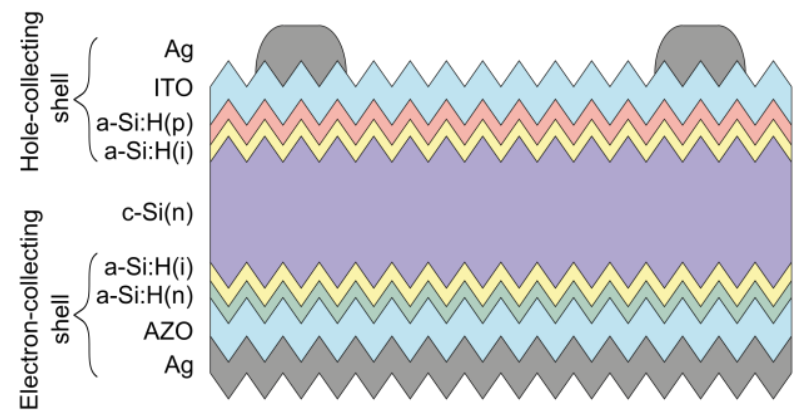

Fig.1. Schematic cross-section of the monofacial SHJ solar cell architecture with front hole-collecting shell and an AZO layer in the rear electron-collecting shell.

TABLE I

AZO LAYER PARAMETERS.

\begin{tabular}{ccccc}
\hline \hline Layer ID & $\mathrm{O}_{2}(\%)$ & $\mathrm{t}(\mathrm{s})$ & $\mathrm{N}\left(\mathrm{cm}^{-3}\right)$ & $\mathrm{w}(\mathrm{nm})$ \\
\hline$\# 1$ & 0.07 & 250 & $1.94 \times 10^{20}$ & 160 \\
$\# 2$ & 0.10 & 250 & $9.37 \times 10^{19}$ & 160 \\
$\# 3$ & 0.15 & 250 & $4.08 \times 10^{19}$ & 160 \\
$\# 4$ & 0.15 & 100 & $9.45 \times 10^{19}$ & 65 \\
$\# 5$ & 0.15 & 170 & $6.16 \times 10^{19}$ & 105 \\
$\# 6$ & 0.15 & 390 & $3.90 \times 10^{19}$ & 245 \\
$\# 7$ & 0.15 & 620 & $2.39 \times 10^{19}$ & 380 \\
\hline \hline
\end{tabular}

$\mathrm{O}_{2}$ : oxygen content in the AZO deposition gas flow; t: deposition time; $\mathrm{N}$ : carrier concentration; w: layer thickness (on glass).

\section{RESULTS AND DISCUSSION}

\section{A. Light Management}

The $\mathrm{J}_{\mathrm{SC}}$ of solar cells with different oxygen content in the AZO deposition gas flow and a fixed thickness of $160 \mathrm{~nm}$ are depicted in Figure 2. First, it is observed that the $\mathrm{J}_{\mathrm{SC}}$ strongly increases with the oxygen content. Namely, increasing the $\mathrm{O}_{2}$ content in the deposition gas flow from 0.07 to $0.15 \%$ increases the $\mathrm{J}_{\mathrm{SC}}$ by $0.56 \mathrm{~mA} / \mathrm{cm}^{2}$. Consistently, the corresponding $\mathrm{EQE}$ curves (see Figure 4) are higher in the $1000 \mathrm{~nm}$ to $1200 \mathrm{~nm}$ spectral range for higher oxygen contents. Similarly to what was observed in [6] for the case of ITO, the reflectivity value at $1200 \mathrm{~nm}$ (see also Figure 4) increases for higher oxygen contents (lower carrier concentrations). Overall, it is thus observed that the increase in oxygen content reduces the parasitic absorption in the AZO layer, as evidenced by the decrease in the 1-R-EQE curves (Figure 4). Hence, decreasing the AZO carrier concentration consistently reduces the FCA losses, resulting in an improved IR back reflector (higher escape light) allowing higher $\mathrm{J}_{\mathrm{SC}}$.

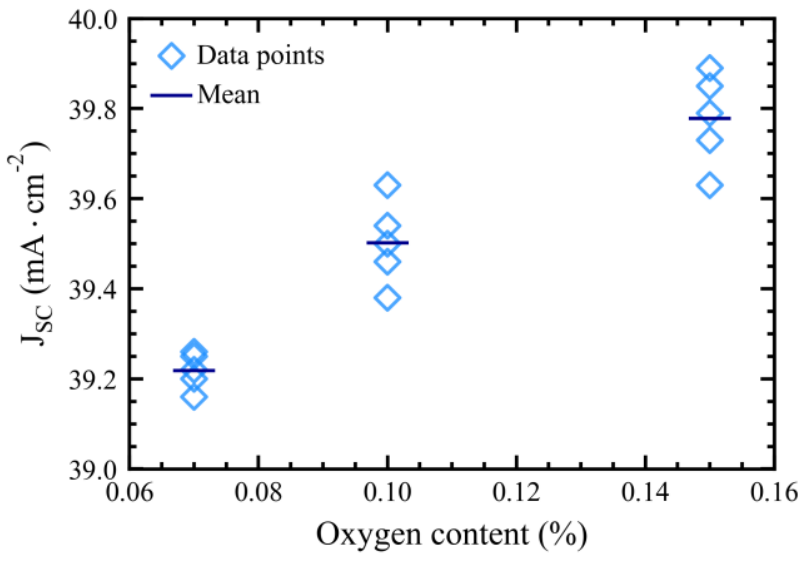

Fig. 2. $\mathrm{J}_{\mathrm{SC}}$ of co-deposited $4-\mathrm{cm}^{2}$ solar cells with varying $\mathrm{O}_{2}$ content in the AZO deposition gas flow. The AZO thickness is $160 \mathrm{~nm}$ for all the devices. The black dash represents the mean value over the 5 cells, each represented by the blue data points.

The $\mathrm{J}_{\mathrm{SC}}$ of cells with different AZO thicknesses and a constant $\mathrm{O}_{2}$ content of $0.15 \%$ are depicted in Figure 3. A J $\mathrm{JC}_{\mathrm{SC}}$ gain of $0.23 \mathrm{~mA} / \mathrm{cm}^{2}$ is obtained when replacing the $65-\mathrm{nm}$ thick AZO layer by a $380-\mathrm{nm}$-thick one. Compared to the oxygen series, this means that an additional $0.11 \mathrm{~mA} / \mathrm{cm}^{2}$ gain with respect to the $160 \mathrm{~nm}$ and $0.15 \%$ AZO layer could be obtained. Consistently, the EQE, 1-R (total absorption) and 1R-EQE (parasitic absorption) curves in Figure 4 show the lowest IR parasitic absorption losses in the 1000 to $1200 \mathrm{~nm}$ range for the device with a 380-nm-thick rear AZO layer. As a first conclusion we can hence state that this additional $\mathrm{J}_{\mathrm{SC}}$ gain comes from a reduction of the plasmonic absorption as the thickness increases, since fewer evanescent waves reach the blanket silver layer.

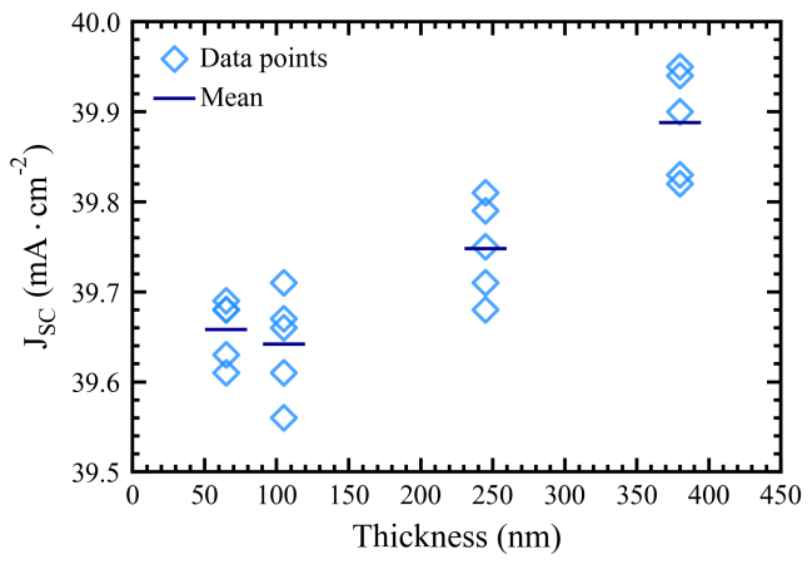

Fig. 3. $\mathrm{J}_{\mathrm{SC}}$ of co-deposited $4-\mathrm{cm}^{2}$ solar cells with varying AZO thickness. The $\mathrm{O}_{2}$ content in the AZO deposition gas flow is $0.15 \%$ for all the devices. The black dash represents the mean value over the 5 cells, each represented by the blue data points. 


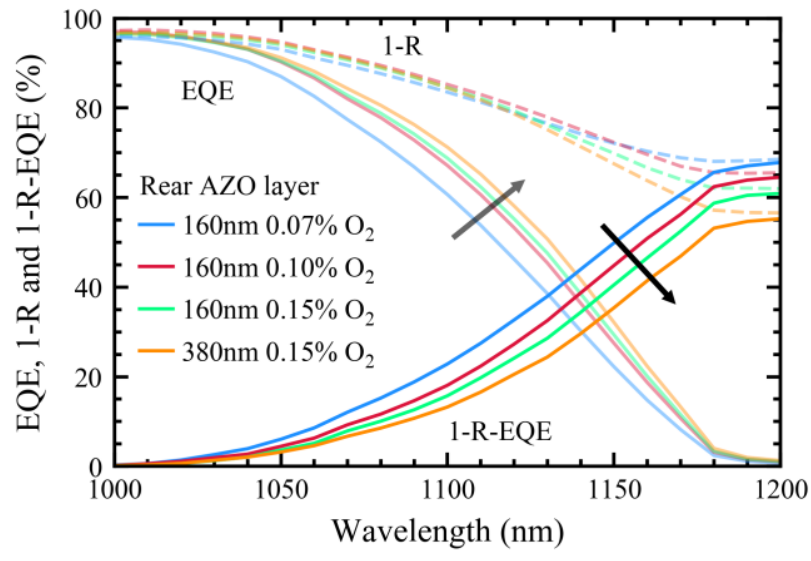

Fig. 4. EQE (solid light), 1-R (dashed light) and 1-R-EQE (solid) of codeposited $4-\mathrm{cm}^{2}$ solar cells with varying $\mathrm{O}_{2}$ content in the AZO deposition gas flow for a fixed thickness of $160 \mathrm{~nm}$ along with the highest thickness of $380 \mathrm{~nm}$ with $0.15 \%$ of $\mathrm{O}_{2}$ content. The black arrows provide a visual guide to highlight the increase of EQE and decrease of total parasitic absorption with respect to the considered layers.

However, the measurements given in Table I reveal that, for a given oxygen content, the carrier concentrations of the investigated AZO layers decrease when their thickness increases (see layers \#4 to \#7 in Table I). This may be due to a higher defect density in the vicinity of the substrate interface induced by the nucleation layer of the TCO. Such defects, like oxygen vacancies, can act as donor levels hence increasing the carrier concentration. Such a dependence of the carrier concentration to the thickness has already been observed in [6] for the case of ITO layers, and further material analysis are proposed in [26]. Therefore, the carrier concentration within the AZO layer and its thickness cannot be considered as two independent parameters. To be free of this bias, it is more accurate to investigate the $\mathrm{J}_{\mathrm{SC}}$ as a function of $D$, the total number of carriers per unit area, hereafter simply calculated as the carrier concentration multiplied by the layer thickness: $D=$ $N \cdot w$. This quantity is directly related to FCA as the larger the carrier concentration or the thickness (i.e. the larger D), the larger the FCA. Note that the optical mobility is expected to be affected by the carrier concentration through impurity scattering. Higher carrier concentrations will lead to lower optical motilities. However the change will be of a much smaller magnitude compared to the carrier concentration variation in the investigated range of this paper. We expect about $20 \%$ change of optical mobility for an order of magnitude change in carrier concentration [27]. Both effects will affect the FCA in the same direction; however the dominant effect will be carrier concentration. The data of Figure 2 and Figure 3, as well as several additional data for the oxygen content series, are plotted in Figure 5 as a function of $D$. With such a representation, it is possible to calculate and decouple the $\mathrm{J}_{\mathrm{SC}}$ gain owing to the FCA reduction and the one owing to the plasmonic absorption reduction. The blue data points in Figure 5 represent the $\mathrm{J}_{\mathrm{SC}}$ variation for a fixed AZO thickness of about $160 \mathrm{~nm}$ but a change of carrier concentration from $2.6 \times 10^{19}$ to $2.0 \times 10^{20} \mathrm{~cm}^{-3}$. Here, the $\mathrm{J}_{\mathrm{SC}}$ steadily increases when $D$ decreases. This is clearly related to the FCA reduction associated to the carrier concentration decrease. In contrast, the red data points represent the thickness series from
65 to $380 \mathrm{~nm}$ (see layers \#4 to \#7 in Table I). For these layers, a $\mathrm{J}_{\mathrm{SC}}$ gain of $0.25 \mathrm{~mA} / \mathrm{cm}^{2}$ is observed for similar values of $D$, i.e. for the same amount of FCA losses. Hence, this gain is the result of the plasmonic absorption reduction. Overall, reduction in the plasmonic absorption losses obtained with thicker AZO layers is larger than the additional FCA losses implied by thicker AZO layer, eventually leading to a $\mathrm{J}_{\mathrm{SC}}$ increase.

Note that, as commonly observed for TCOs, the refractive index of AZO in the IR spectral range is correlated to its carrier concentration: the lower the carrier concentration, the higher the refractive index. Hence, the Fresnel coefficients at the c$\mathrm{Si} / \mathrm{TCO}$ and the TCO/metal interfaces will change with the TCO carrier concentration. The consequences for FCA and plasmonic absorption losses have been thoroughly investigated in [6] in the case of ITO, and it was shown that for most of the incident angles, absorption losses decrease when the TCO carrier concentration decreases. Therefore, potential IR reflection gains from lower TCO refractive index never offset FCA losses and the IR EQE always benefits from a reduction of the TCO carrier concentration, as we observe for AZO here.

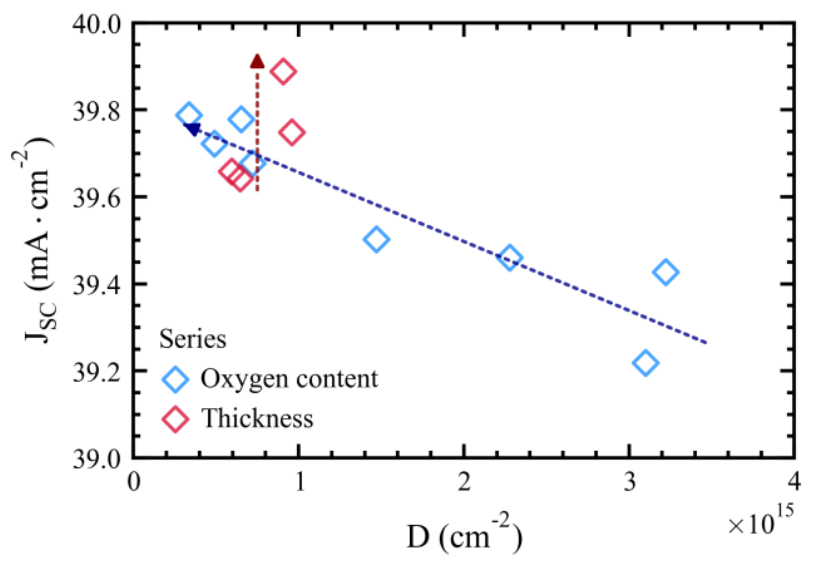

Fig. 5. $\mathrm{J}_{\mathrm{SC}}$ as a function of $D$, the total number of carriers per unit area, for different AZO layers. Each data point is the $\mathrm{J}_{\mathrm{SC}}$ value averaged over 5 devices. The oxygen content variation is represented by the blue data points and the thickness variation by the red data points. Two arrows highlight the $\mathrm{J}_{\mathrm{SC}}$ gain which owes to the FCA decrease (blue dashed arrow) and to the plasmonic absorption decrease (red dashed arrow).

\section{B. Transport Losses}

As mentioned above in Section I, the $\mathrm{J}_{\mathrm{SC}}$ improvement must not jeopardize the electrical properties of the final solar cells. In this part of the paper, the $\mathrm{pFF}, \mathrm{FF}, \mathrm{V}_{\mathrm{OC}}$ and $\mathrm{R}_{\mathrm{S}}$ values of our cells are investigated. Figure 6 plots the pFF, FF and $\mathrm{V}_{\mathrm{OC}}$ of the thickness series (see layers \#4 to \#7 in Table I). First, it is observed that the $\mathrm{pFF}$ and FF values are not directly affected by the rear AZO thickness in the investigated range, even if they present some fluctuations. No monotonic variation of the FF as a function of the thickness is observed. Instead, the FF values fluctuate by about $\pm 0.7 \%$ abs around a mean value of $79.5 \%$. The FF values follow the same fluctuation trends as the $\mathrm{pFF}$ ones, which means that the $\mathrm{R}_{\mathrm{S}}$ is not actually affected in the investigated range of thickness. We hypothesize that this scattering is the consequence of the wafer-to-wafer variability within a single batch of devices rather than a sign of impeded carrier transport. The $V_{O C}$ values presented in Figure 6 follow the same variations as FF ones with fluctuations of about $\pm 3 \mathrm{mV}$ around a mean value of $727 \mathrm{mV}$. We assume again that 
this scattering is due to the consequence of the wafer-to-wafer

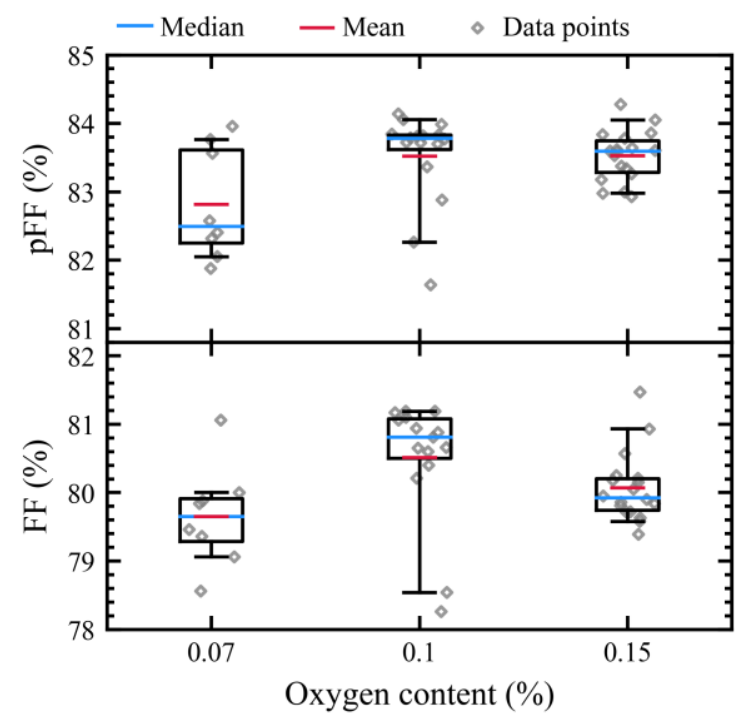

fluctuations with a standard deviation between 0.5 to $0.9 \%$ abs

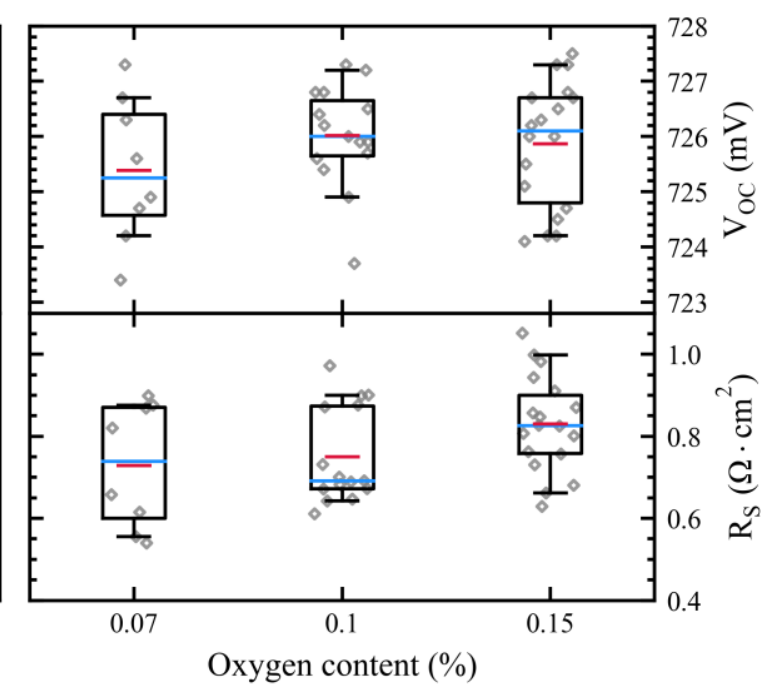

Fig. 7. Box plots of $\mathrm{pFF}, \mathrm{FF}, \mathrm{V}_{\mathrm{OC}}$ and $\mathrm{R}_{\mathrm{S}}$ values of $4-\mathrm{cm}^{2}$ solar cells for several runs. The oxygen content in the AZO deposition gas flow varies from 0.07 to $0.15 \%$ and the thickness is kept constant around $160 \mathrm{~nm}$ with a variation of about $9.4 \%$. The boxes are delimited by the first and the third quartiles. The median is represented by the blue line and the mean by the red line. The whiskers mark the 5 and 95 percentiles.

variability within a single batch of devices rather than the sign of a direct change in the passivation or selectivity of the devices. Consequently, the $\mathrm{J}_{\mathrm{SC}}$ gain seen in Figure 3 eventually drives the efficiency increase, leading up to $0.22 \%$ abs Eff. gain when replacing the 65 -nm-thick AZO layer by a 380 -nm-thick one.

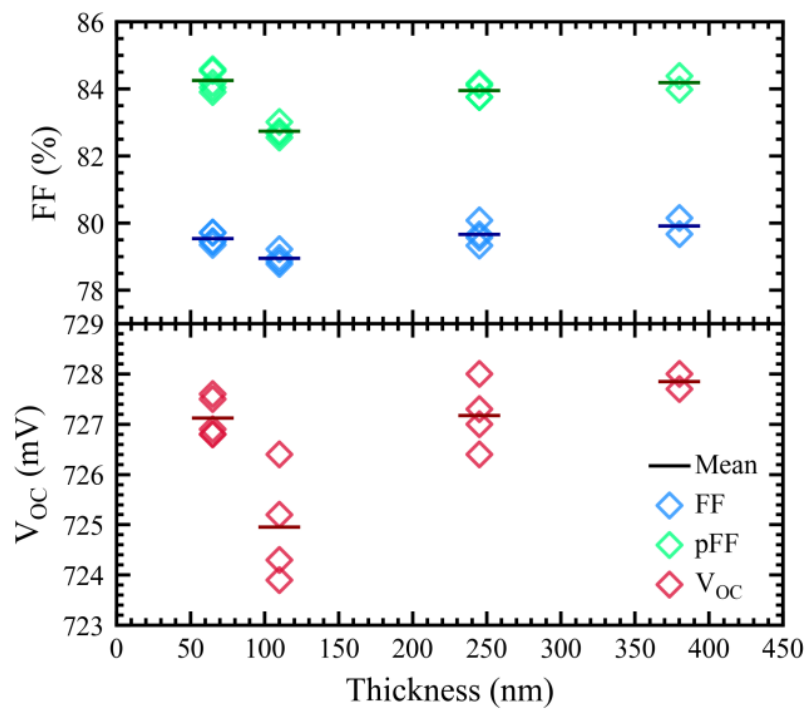

Fig. 6. pFF, $\mathrm{FF}$ and $\mathrm{V}_{\mathrm{OC}}$ values of co-deposited $4-\mathrm{cm}^{2}$ solar cells with varying AZO thickness (cf. layers \#4 to \#7 in Table I). The $\mathrm{O}_{2}$ content in the AZO deposition gas flow is $0.15 \%$ for all devices. The black dash represents the average value over 2 to 5 cells represented by the data points.

Regarding the oxygen content variation, several runs were performed to study and validate the behavior of $\mathrm{FF}$ and $\mathrm{V}_{\mathrm{OC}}$ over the AZO oxygen content range under investigation for a thickness of about $160 \mathrm{~nm}$. Figure 7 depicts the pFF, FF, $V_{O C}$ and $\mathrm{R}_{\mathrm{S}}$ values over 0.07 to $0.15 \%$ of $\mathrm{O}_{2}$ range, corresponding to a carrier concentration variation from $4.1 \times 10^{19}$ to $2.0 \times 10^{20} \mathrm{~cm}^{-3}$. Firstly, it is observed that FF values present which lies within the typical dispersion of our process and material. The median values for the extremes $\mathrm{O}_{2}$ contents $(0.07$ and $0.15 \%$ ) are close to each other, just below $80 \%$. However, a slight FF optimum seems to exist for $0.10 \% \mathrm{O}_{2}$ content, resulting in a higher median FF value of $80.8 \%$. This optimum owes to the $\mathrm{pFF}$ and $\mathrm{R}_{\mathrm{S}}$ behaviors. Indeed, the median $\mathrm{pFF}$ for the $0.07 \% \mathrm{O}_{2}$ content is $82.5 \%$, increases to $83.8 \%$ for the $0.10 \% \mathrm{O}_{2}$ content, then stabilizes at $83.6 \%$ for $0.15 \% \mathrm{O}_{2}$ content. Likewise, the $\mathrm{V}_{\mathrm{OC}}$ values exhibit a similar behavior, going from a median value of $725 \mathrm{mV}$ for the $0.07 \% \mathrm{O}_{2}$ content up to $726 \mathrm{mV}$ for both the 0.10 and $0.15 \% \mathrm{O}_{2}$ contents. Such observations suggest a better c-Si passivation which may owe to a change of field effect passivation provided by variations in the AZO layer properties. However, the precise physical phenomena explaining this behavior remain unclear. Secondly, the $\mathrm{R}_{\mathrm{S}}$ values present similar median values for the 0.07 and $0.10 \% \mathrm{O}_{2}$ contents, namely 0.74 and $0.69 \Omega \cdot \mathrm{cm}^{2}$ respectively, but a higher value of $0.83 \Omega \cdot \mathrm{cm}^{2}$ for the $0.15 \% \mathrm{O}_{2}$ content. Consequently, the combined high $\mathrm{pFF}$ and low $\mathrm{R}_{\mathrm{S}}$ values of the $0.10 \% \mathrm{O}_{2}$ content lead to the observed FF optimum. The two other $\mathrm{O}_{2}$ contents present either a low $\mathrm{pFF}$ with a low $\mathrm{R}_{\mathrm{S}}$ value $\left(0.07 \% \mathrm{O}_{2}\right.$ content $)$ or a high $\mathrm{pFF}$ with a high Rs value $(0.15 \%$ $\mathrm{O}_{2}$ content), explaining their lower FF values. Overall, as the electrical properties are only marginally impacted in the oxygen range investigated here, the $\mathrm{J}_{\mathrm{SC}}$ gain seen in Figure 2 eventually drives the efficiency increase, leading Eff. up to $0.54 \%$ abs gain when replacing the $0.07 \% \mathrm{O}_{2}$ content AZO layer by a $0.15 \%$ $\mathrm{O}_{2}$ content one.

The observed changes in passivation and $\mathrm{R}_{\mathrm{S}}$ with the changes of oxygen content in the investigated AZO layers can be due to several phenomena: (i) a modification of the TCO bulk resistivity or (ii) a modification of the AZO properties, such as its workfunction or a change in its structural material properties affecting the band alignment within the whole electroncollecting shell. This can affect both the contact resistance of 
the rear shell as well as its passivation properties. Regarding (i), as the devices under investigation are in monofacial configuration, the rear TCO bulk resistivity change will only affect the vertical transport and thus has a negligible effect on $\mathrm{R}_{\mathrm{S}}$. The additional $\mathrm{R}_{\mathrm{S}}$ induced by the rear TCO bulk resistivity change were calculated to be well below $1 \mu \Omega \cdot \mathrm{cm}^{2}$. Considering that a $1 \Omega \cdot \mathrm{cm}^{2} \mathrm{R}_{\mathrm{S}}$ induces a FF loss of $\sim 5 \%$ abs [25], such low equivalent $\mathrm{R}_{\mathrm{S}}$ values obviously have an insignificant impact on the actual FF variation of the devices. Regarding (ii), it is known from the literature that the properties of the thin silicon layers, the TCO and their coupling define the global transport mechanisms inside the whole shell [28], [29]. More specifically, for an electron-collecting shell, recent simulations show that a higher carrier concentration of the TCO - therefore a lower workfunction - is beneficial to the carrier transport, as it promotes several transport mechanisms such as thermionic emission, direct tunneling and trap-assisted tunneling [30]. Therefore, lowly-doped AZO should be detrimental to carrier transport which is not observed in our results presented in Figure 7. For these reasons, further investigation are required to verify our observations and hypotheses. More precisely, the investigation of the AZO properties, and particularly how its carrier concentration affects the band structure of the whole electron-collecting shell, is necessary to prove if the hypothesis (ii) is the origin of the FF optimum. This requires to investigate how the transport losses, encompassing passivation and series resistance (including selectivity), are modified as the AZO layers are optimized. This is out of the scope of this paper and will be the topic of a future publication.

\section{Outlook}

In the course of our investigations, the 160-nm-thick AZO layer with $0.10 \% \mathrm{O}_{2}$ content was integrated in the rear reflector of $4-\mathrm{cm}^{2}$ monofacial solar cells (wafer thickness of $240 \mathrm{um}$ ) with front hole-collecting shell and about $3.25 \%$ optical shadowing. This led to a champion device featuring an Eff. of $23.96 \%$ (Figure 8). Note that this champion devices features improved thin silicon and TCO layers compared to the ones used for our optimization study presented above. A $\mathbf{J}_{\mathrm{SC}}$ of $40.81 \mathrm{~mA} / \mathrm{cm}^{2}$ was obtained, without compromising the $\mathrm{V}_{\mathrm{OC}}$ and the FF, which present good values of $726 \mathrm{mV}$ and $80.87 \%$, respectively. This full AZO rear reflector features a gain of $0.3 \mathrm{~mA} / \mathrm{cm}^{2}$ in $\mathrm{J}_{\mathrm{SC}}$ compared to our baseline back ITO reported in [21]. AZO thus demonstrates a clear potential to act as a high quality indium-free back TCO in rear electron-collecting shells to reach almost $24 \%$ efficiency. As presented in section III.A and III.B, even higher solar cells performances are within reach as the rear AZO layer can be further optimized through its thickness (see in Figure 3 and Figure 6 the 380-nm-thick AZO layer) as well as through a more careful refining of its oxygen content. Moreover, the outcomes of our study remain valid in the case of bifacial solar cells, which are the current trend for SHJ solar cells mass production. Indeed, in such devices, the full rear silver blanket layer is replaced by a dense screenprinted silver grid: FCA and plasmonic absorption are therefore still present - yet with different proportions compared to monofacial solar cells - and our parasitic absorption mitigation approach can be equally applied.

In a more global perspective, each TCO (ITO and AZO being two prominent examples) can in principle be optimized to meet the required trade-off between FCA and plasmonic absorption losses mitigation to achieve higher $\mathrm{J}_{\mathrm{SC}}$. However, as presented in section I, because of the existing trade-off between the electrical and the optical properties of the shells, the electrical performances of these TCO must be well controlled alongside their optical ones in order to convert the $\mathrm{J}_{\mathrm{SC}}$ gain into higher solar cell efficiency. Eventually, the best TCO will then be the one, which, once integrated in the shell, allows the highest light collection (highest $\mathrm{J}_{\mathrm{SC}}$ ) while keeping the highest electrical performances (i.e. high $\mathrm{V}_{\mathrm{OC}}$ and $\mathrm{FF}$ ). For these reasons, to go further in the understanding of impact of the TCO properties (when optimizing the light collection) on the global transport losses of back electron-collecting shells, measurements of the layers characteristics related to selectivity and passivation are ongoing and will be the subject of another paper. More precisely, the TCO carrier concentration and thickness, the crystallinity, thickness and activation energy of the thin silicon layers (amorphous or micro-crystalline) used in the shells, as well as their contact resistance to the TCO, are expected to play a major role [16][17]. This study will eventually define the best TCO candidate for back electron-collecting shells.

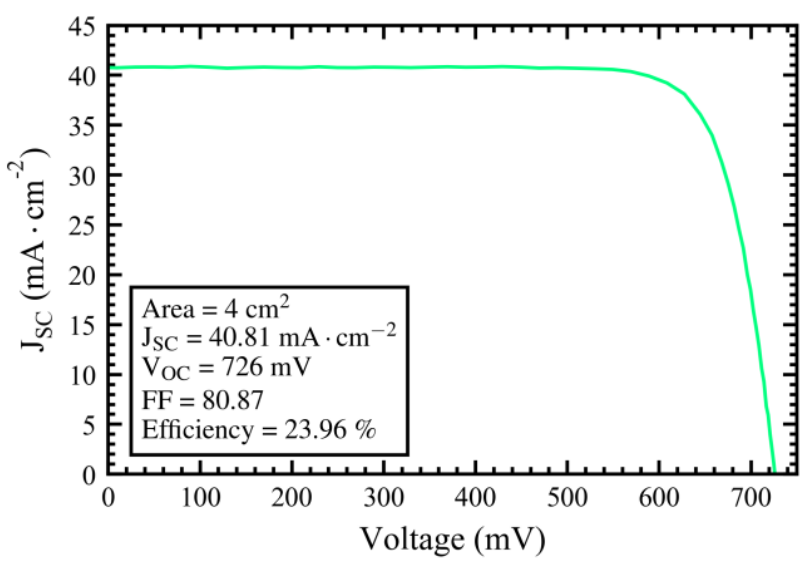

Fig. 8. Current-voltage characteristic of the best HJT 4- $\mathrm{cm}^{2}$ cells integrating AZO rear reflector with $0.10 \% \mathrm{O}_{2}$ content and $160 \mathrm{~nm}$ (in-house measurement).

\section{CONCLUSION}

In this contribution, the flexibility of the rear AZO layer to be tuned over a wide range of oxygen contents and thicknesses to improve the IR-response of $4 \mathrm{~cm}^{2}$ monofacial solar cells with front hole-collecting shells has been demonstrated. The rear AZO layer can be optimized to behave as an optical layer which minimizes free-carrier and plasmonic absorptions in addition to its primary role as electrical contact layer inside the rear electron-collecting shell. Overall, looking at the extreme conditions for thickness and oxygen series, it is demonstrated that the electrical properties are not jeopardized by the increase in thickness as well as by the decrease in the carrier concentration over the investigated ranges. Based on our experiments, the electrical properties are even slightly improved for some specific layer conditions. The $\mathrm{J}_{\mathrm{SC}}$ improvement due to the important FCA and plasmonic absorption decrease combined with the good electrical properties of the rear electron-collecting shell eventually leads to an efficiency up to $23.96 \%$. Finally considering the concerns regarding the indium availability in the case of a large scale 
deployment of the SHJ technology, AZO is an interesting and promising candidate to replace ITO at a potentially lower cost [31].

\section{ACKNOWLEDGEMENTS}

This project has received funding from the European Union's Horizon 2020 research and innovation programme under grant agreement $\mathrm{N}^{\circ} 745601$.

\section{REFERENCES}

P. Würfel, Physics of Solar Cells: From Principles to New Concept. 2008.

[2] A. Cuevas, T. Allen, J. Bullock, W. Yimao, D. Yan, and X. Zhang, "Skin care for healthy silicon solar cells," 2015 IEEE 42nd Photovolt. Spec. Conf., pp. 1-6, 2015.

[3] A. Fell, J. Schön, M. C. Schubert, and S. W. Glunz, "The concept of skins for silicon solar cell modeling," Sol. Energy Mater. Sol. Cells, vol. 173, pp. 128-133, 2017.

[4] A. Cuevas et al., "Carrier population control and surface passivation in solar cells," Sol. Energy Mater. Sol. Cells, vol. 184, pp. 38-47, 2018.

[5] M. Taguchi et al., " $24.7 \%$ Record efficiency HIT solar cell on thin silicon wafer," IEEE J. Photovoltaics, vol. 4, no. 1, pp. 96-99, 2014.

[6] Z. C. Holman et al., "Infrared light management in high-efficiency silicon heterojunction and rear-passivated solar cells Infrared light management in high-efficiency silicon heterojunction and rearpassivated solar cells," J. Appl. Phys., vol. 113, no. 1, p. 013107 , 2013.

[7] Z. C. Holman, A. Descoeudres, S. De Wolf, and C. Ballif, "Record infrared internal quantum efficiency in silicon heterojunction solar cells with dielectric/metal rear reflectors," IEEE J. Photovoltaics, vol. 3, no. 4, pp. 1243-1249, 2013.

[8] Z. C. Holman, S. De Wolf, and C. Ballif, "Improving metal reflectors by suppressing surface plasmon polaritons: A priori calculation of the internal reflectance of a solar cell," Light Sci. Appl., vol. 2, no. e106, pp. 1-6, 2013.

[9] S. Kim et al., "Surface-plasmon-induced light absorption on a rough silver surface," vol. 011109, no. December 2010, pp. 1-4, 2011. K. L. Chopra, "Transparent conductors - A status review," Thin Solid Films, vol. 102, no. 1, pp. 1-46, 1983.

[11] B. G. Lewis and D. C. Paine, "A pplications and Processing of Transparent Oxides," MRS Bull., vol. 25, no. 8, pp. 22-27, 2000. K. Ellmer, "Resistivity pf polycristalline zinc oxide films: current status and physical limit," J. Phys. D. Appl. Phys., vol. 34, pp. 3097-3108, 2001.

[13] S. De Wolf, A. Descoeudres, Z. C. Holman, and C. Ballif, "Highefficiency Silicon Heterojunction Solar Cells: A Review," Green, vol. 2, no. 1, pp. 7-24, 2012.

[14] C. Battaglia, A. Cuevas, and S. De Wolf, "High-efficiency crystalline silicon solar cells: Status and perspectives," Energy Environ. Sci., vol. 9, no. 5, pp. 1552-1576, 2016.

[15] L-.L. Senaud et al., "High efficiency silicon heterojunction solar cells with improved IR response," EUPVSEC, 2017.

[16] P. Procel, G. Yang, O. Isabella, and M. Zeman, "Theoretical evaluation of contact stack for high efficiency IBC-SHJ solar cells," Sol. Energy Mater. Sol. Cells, vol. 186, pp. 66-77, 2018.

[17] M. Bivour, M. Reusch, F. Feldmann, M. Hermle, and S. W. Glunz, "Requirements for Carrier Selective Silicon Heterojunctions," Proc. 24th Work. Cryst. Silicon Sol. Cells Modul., no. July, pp. 1-9, 2014.

[18] Gabriel Christmann et al., "Silicon heterojunction solar cells using aluminium doped zinc oxide as back contact sputtering and ALD," EUPVSEC, 2016.

[19] A. B. Morales-vilches et al., "ITO-Free Silicon Heterojunction Solar Cells With $\mathrm{ZnO}: \mathrm{Al} / \mathrm{SiO} 2$ Front Electrodes Reaching a Conversion Efficiency of $23 \%$," IEEE J. Photovoltaics, pp. 1-6, 2018.

[20] A. Descoeudres et al., "Improved amorphous/crystalline silicon interface passivation by hydrogen plasma treatment," Appl. Phys. Lett., vol. 99, no. 12, pp. 1-4, 2011.

[21] A. Descoeudres et al., "Advanced Silicon Thin Films for High-
Efficiency Silicon Heterojunction-Based Solar Cells," 2017 IEEE 44th Photovolt. Spec. Conf. (PVSC), Washington, D.C., USA, pp. 50-55, 2017.

[22] R. A. Sinton, A. Cuevas, and M. Stuckings, "Quasi-steady-state photoconductance, a new method for solar cell material and device characterization," Conf. Rec. Twenty Fifth IEEE Photovolt. Spec. Conf. - 1996, pp. 457-460, 1996.

[23] D. C. Paine, B. Yaglioglu, J. Berry, and D. S. Ginley, Handbook of Transparent Conductors. Chapter 4: Characterization of TCO Materials. Media, Springer Science \& Business, 2010.

[24] S. Bowden and A. Rohatgi, "Rapid and accurate determination of series resistance and fill factor losses in industrial silicon solar cells," 17th Eur. Photovolt. Sol. Energy Conf., 2001.

[25] D. Pysch, A. Mette, and S. W. Glunz, "A review and comparison of different methods to determine the series resistance of solar cells," Sol. Energy Mater. Sol. Cells, vol. 91, pp. 1698-1706, 2007.

[26] D. C. Look et al., "Model for thickness dependence of mobility and concentration in highly conductive zinc oxide concentration in highly conductive zinc oxide," Opt. Eng., vol. 52, pp. 1-5, 2013.

[27] J. Steinhauser, S. Faÿ, N. Oliveira, E. Vallat-Sauvain, and C. Ballif, "Transition between grain boundary and intragrain scattering transport mechanisms in boron-doped zinc oxide thin films," Appl. Phys. Lett., vol. 9, no. 142107, pp. 1-3, 2007.

[28] T. F. Schulze, L. Korte, E. Conrad, M. Schmidt, and B. Rech, "Electrical transport mechanisms in a - Si : H / c -Si heterojunction solar cells," no. 107, pp. 1-13, 2010.

[29] R. Varache, "Développement, caractérisation et modélisation d'interface pour cellules solaires à haut rendement à base d'hétérojunctions de silicium," Université Paris Sud - Paris XI, 2012.

[30] P. Procel, "Understanding the Effect of Hetero-Interfaces Transport on Contact Resistance for c-Si Carrier Selective Contacts," SiliconPV, 2019.

[31] A. Louwen, W. Van Sark, R. Schropp, and A. Faaij, “A cost roadmap for silicon heterojunction solar cells," Sol. Energy Mater. Sol. Cells, vol. 147, pp. 295-314, 2016.

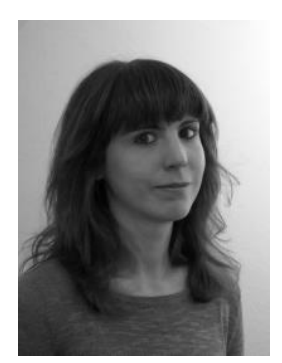

Laurie-Lou Senaud received her M.Sc. degree in Applied Physics from École Polytechnique Fédérale de Lausanne (EPFL), Lausanne, Switzerland, in 2016. Her curriculum was oriented towards theoretical physics with an additional specialization in renewable energies. Her Master thesis was carried out on advanced architectures for competitive high performance crystalline silicon photovoltaics at PV-Center, Centre Suisse d'Électronique et de Microtechnique (CSEM), Neuchâtel, Switzerland. Since 2017 she has been working toward the Ph.D. degree with the PV-Center at CSEM. Her current research interests revolve around electrical transport losses mitigation including contact characterization and improvements for high efficiency crystalline silicon solar cells.

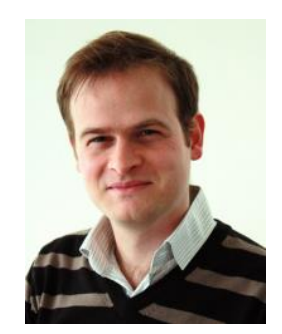

Gabriel Christmann received two master degrees in 2004, one in physical engineering from ESPCI Paris and one in solid state physics from Université Pierre et Marie Curie, Paris, France. From 2004 to 2008 he was a PhD student in the laboratory of advanced semiconductors for photonics and electronics at École polytechnique fédérale de Lausanne where he was awarded a $\mathrm{PhD}$ in photonics in 2009. He was working on light matter coupling at room temperature in wide bandgap semiconductors. He was then a research associate from 2008 to 2013 in the Nanophotonics 
Centre of the Cavendish Laboratory, University of Cambridge, UK. He studied there Bose-Einstein condensation and superfluidity of microcavity polaritons. After a 5 month stay in the biotech startup company Base4 in Cambridge UK, he was a PostDoctoral researcher from 2013 to 2014 in the Microelectronic Research Group, at FORTH/IESL and University of Crete, Heraklion, Greece, where he worked on photonic devices based on polariton physics. He finally joined in 2015 the PV-Center, Centre Suisse d'Électronique et de Microtechnique, Neuchâtel, Switzerland. His research interests include novel contacting schemes for high efficiency solar cells.

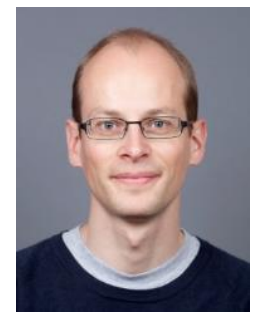

Antoine Descoeudres received the M.Sc. and Ph.D. degrees in physics from Ecole Polytechnique Fédérale de Lausanne, Switzerland, in 2001 and 2006, respectively. From 2007 to 2009, he was a Postdoctoral Researcher at CERN, Geneva, Switzerland, studying vacuum breakdowns for the development of high-gradient accelerating cavities. From 2009 to 2013, he was a Postdoctoral Researcher at the Photovoltaics and Thin-Film Electronics Laboratory, Ecole Polytechnique Fédérale de Lausanne, Neuchâtel, Switzerland, working on the silicon heterojunction technology. In 2013, he joined the PV-center, Centre Suisse d'Electronique et de Microtechnique, Neuchâtel, Switzerland, where he is responsible for the development of silicon heterojunction solar cells. His current research interests include thin-film deposition with plasma-enhanced chemical vapor deposition for high-efficiency crystalline silicon solar cells.

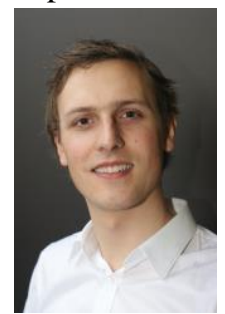

Jonas Geissbühler received a Bachelor degree in micro and nanosciences from the University of Neuchatel and a master degree in micro-engineering at the Ecole Polytechnique Fédérale de Lausanne (EPFL) in 2011. He received his Ph.D. in 2015 from EPFL writing his thesis on high-efficiency silicon heterojunction solar cells. In 2016, he joined the CSEM PV-Center. His research interests include the development of high efficiency silicon heterojunction solar cells.

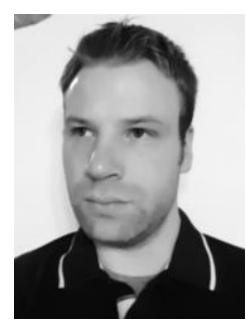

Loris Barraud received his Diplôme HES in microtechnics from HES-SO, Haute école spécialisée, Suisse-Ouest, switzerland, in 2009. From 2009 to 2013 he worked at the Photovoltaics and Thin-Film Electronics Laboratory, École polytechnique fédérale de Lausanne, Neuchâtel, Switzerland, working as deposition engineer with the focus on optoelectronical coatings for silicon heterojunction solar cells. From 2013 to 2018 he worked at the PV-Center, Centre Suisse d'Électronique et de Microtechnique, Neuchâtel, Switzerland, with the focus on developing high efficiency silicon solar cell. Since 2018, he joined the research and development lab of Indeotec SA, Neuchatel, Switzerland.

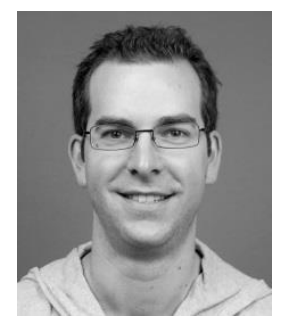

Nicolas Badel obtained a certificate of capacity after a professional training at the école polytechnique fédérale de Lausanne (EPFL) to become laboratory technician in chemistry, in 2001. He joined the team of a start-up company and participated to the development and the industrialization of solid oxide fuel cells (SOFC) within the company HTceramix SA, until 2009. From 2010 to 2012, he worked on the improvement of flexible solar panels packaging, in the R\&D Back End department of VHFTechnologies SA. In 2012, Mr Badel integrated the photovoltaics and thin film laboratory (PV-Lab) of Prof. Christophe Ballif in the institute of microengineering (IMT) and started at the new photovoltaic division of the Centre Suisse d'Electronique et de Microtechnique SA (CSEM), Neuchâtel, in 2013. His work is focusing on the development of different metallization technics for silicon heterojunction solar cells and more specifically the screen-printing method.

Christophe Allebé received his master degree in Physics from the Université Catholique de Louvain. From 2000 to 2011 he was sequentially with Interuniversity Microelectronics Center, Leuven, and its spin-off Photovoltech, focusing on crystalline silicon based homo-junction solar cells. He switched then to Sibased hetero-junction solar cells while working for Kaneka, Japan. He joined in 2013 the PV-center at CSEM, where he works on different processes and approaches enabling high solar cells performances.

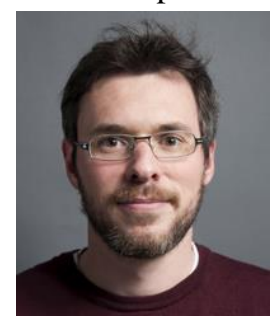

Sylvain Nicolay received the Degree in physics engineering from Ecole Polytechnique, Université Libre de Bruxelles, Brussel, Belgium, in 2003 and the Ph.D. degree in photonics from Ecole Polytechnique Fédérale de Lausanne, Neuchâtel, Switzerland, for work on the development of "III-N heterostructures for intersubband transitions." He then joined the Photovoltaics and Thin-Film Electronics Laboratory, Ecole Polytechnique Fédérale de Lausanne in 2008 to lead the research team working on transparent conductive oxide for photovoltaic (PV) applications. Since 2013, he has been in charge of the coating section with the PV Center, Centre Suisse d'Electronique et de Microtechnique, Neuchâtel.

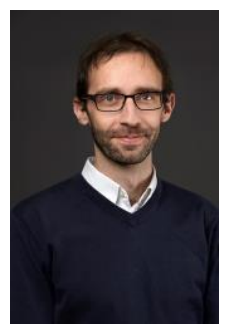

Matthieu Despeisse received his degree in electrical engineering from INSA, Lyon, France, in 2002. He then worked until 2008 at the European Organization for Particle Physics (CERN) in Geneva, Switzerland. His research focused on novel Silicon radiation sensors (3D, SiPM, a-Si:H), advanced microelectronics and technology transfer. $\mathrm{He}$ obtained his $\mathrm{PhD}$ degree in 2006 for his work at CERN on amorphous silicon sensors deposited directly on the readout electronics. He then joined the Photovoltaics and Thin-Film Electronics Laboratory, École polytechnique fédérale de Lausanne, Neuchâtel, Switzerland, in 2009, to lead the research team working on thin film silicon photovoltaics technology. Since 2013, he leads the research activities on crystalline silicon 
photovoltaics in the PV-center, Centre Suisse d'Électronique et de Microtechnique, Neuchâtel, Switzerland, with special focus on silicon heterojunction technology, passivating contacts and metallization.

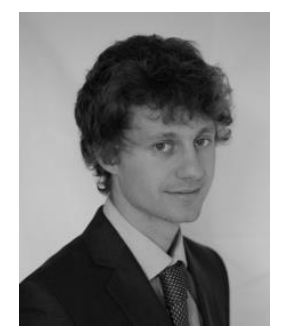

Bertrand Paviet-Salomon was born in Lyon, France, in 1986. He received the M.Sc. degree in 2009 and the Engineer Diploma degree in theoretical and applied optics from the Institut d'Optique, Paris, France. From 2009 to 2012, he was a Ph.D. student with the French National Institute for Solar Energy, Le-Bourget-du-Lac, France, working on laser processes for crystalline silicon solar cells. He received the Ph.D degree in electronics and photonics from the University of Strasbourg, Strasbourg, France, in 2012. From 2012 to 2014, he was a Postdoctoral Researcher at the Photovoltaics and Thin-Film Electronics Laboratory, École polytechnique fédérale de Lausanne, Neuchâtel, Switzerland, working on high-efficiency back-contacted silicon heterojunction solar cells. In 2014, he joined the PV-Center, Centre Suisse d'Électronique et de Microtechnique, Neuchâtel, Switzerland. His research interests include the development of high-efficiency crystalline silicon solar cells.

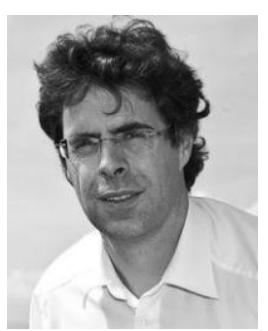

Christophe Ballif received the Graduate's degree in physics and Ph.D. degree from École polytechnique fédérale de Lausanne, Lausanne, Switzerland, in 1994 and 1998, respectively, focusing on novel photovoltaic materials. He was a Postdoctoral Researcher at NREL, Golden, CO, USA. He then was with the Fraunhofer ISE, Freiburg, Germany, where he was involved in crystalline silicon photovoltaics (monocrystalline and multicrystalline) until 2003. He then joined the EMPA, Thun, Switzerland, before becoming a Full Professor and Chair at the Institute of Microengineering, University of Neuchâtel, Switzerland, in 2004. In 2009, the Institute was transferred to EPFL. He is the Director of the Photovoltaics and Thin-Film Electronics Laboratory within the Institute as well as of the CSEM PV-center, dedicated to industrial research and technology transfer. 